\title{
Facteurs limitants et aspects dynamiques de la mycorhization contrôlée de Fagus silvatica Lin. par Hebeloma crustuliniforme (Bull. ex Saint-Amans) Quél. sur tourbe fertilisée
}

\author{
J. GARBAYE et M.E. WILHELM \\ I.N.R.A., Station de Recherche sur les Sols forestiers et la Fertilisation \\ Centre de Recherches forestières de Nancy, Champenoux, F 54280 Seichamps
}

\begin{abstract}
Résumé
Les conditions de la mycorhization du hêtre (Fagus silvatica Lin.) par Hobeloma crustuliniforme (Bull. ex Saint-Amans) Quél. sur tourbe fertilisée ont été étudiées en pépinièrc, en phytotron et in vitro. Deux phases peuvent être distinguées après le semis et l'incorporation de l'inoculum (mycélium inclus dans de la vermiculite) à la tourbe : une première phase de survie puis de disparition du mycélium autour des particules de vermiculite, et une deuxième phase de colonisation de la rhizosphère par le champignon, accompagnée simultanément d'infections primaires puis secondaires. La survie du champignon, pendant la première phase, ne dépend pas de la fertilisation de la tourbe, alors que ce facteur (dose globale et ćquilibre azote/phosphore) est déterminant pendant la deuxième phase. Ces résultats permettent de concevoir une technique efficace d'inoculation en pépinière sur tourbe fertilisée.
\end{abstract}

\section{Introduction}

Parmi les champignons ectomycorhiziens susceptibles d'améliorer la croissance des jeunes plantations de hêtre (Fagus silvalica) dans le nord-est de la France, Hebeloma crustuliniforme s'est avéré intéressant par son efficacité et sa compétitivité (GARBAYE, 1983). Cependant, la maîtrise de son inoculation dans les pépinières sur tourbe fertilisée, de plus en plus utilisée pour la production de plants de hêtre, est apparue difficile dès les premières tentatives (LAINEZ, 1981 ; WILHELM, 1983). Nous avons donc cherché à approfondir notre connaissance du processus d'infection mycorhizienne dans ce cas particulier et recherché les facteurs limitants essentiels du point de vue pratique. L'expérimentation a été menée en pépinière, en phytotron et in vitro sur le champignon seul, en privilégiant l'étude dynamique du phénomène. Tous les essais ont porté sur la tourbe neuve non désinfectée. LAINEz (1981) a en effet montré que la désinfection était nécessaire lorsque la tourbe avait déjà porté une culture de hêtre, à la fois pour favoriser la mycorhization par le champignon introduit et pour éliminer les pathogènes du hêtre (surtout Pythium sp. : Delran et al., 1982). 


\section{Matériel et méthodes}

\subsection{Expérience en pépinière}

Les graines de hêtre de provenance roumaine ont été prégermées 4 semaines à 4 "C dans de la tourbe humide puis semées au printemps à raison de 5 par pot de $9 \mathrm{dm}^{3}$ contenant les substrats étudiés : tourbe blonde de Hollande à différents nivéaux de fertilisation.

Le tableau I donne le délail des 14 traitements réalisés en ne faisant variel qu'un seul facteur à la fois à partir d'un tratement de base commun.

\section{TABLEAU 1}

Plan expérimental de lessai en pépinière.

Design of the nursery experiment.

\begin{tabular}{|c|c|c|}
\hline Facteur & $\begin{array}{l}\text { Traitement } \\
\text { de base }\end{array}$ & Variantes \\
\hline Climat..... & Tunnel ouvert (TO) & $\begin{array}{l}\text { Découvert (D), tunnel fermé (TF), serre } \\
\text { climatisée (S) }\end{array}$ \\
\hline Dose de fertilisation .. & 1 & $1 / 2.2$ \\
\hline $\mathrm{pH}$ & 4 & $5,6,7$ \\
\hline Type d'inoculum & Non lave (NL) & Lavé (1.) \\
\hline $\begin{array}{l}\text { Dose d'inoculum (pour- } \\
\text { centage en volume) . }\end{array}$ & 7 & $1,5,15$ \\
\hline $\begin{array}{l}\text { Répartition de linocu- } \\
\text { lum } \ldots \ldots \ldots \ldots \ldots \ldots\end{array}$ & $\begin{array}{l}\text { Mclangé au substrat } \\
\text { (RM) }\end{array}$ & $\begin{array}{l}\text { Localisé en colonne sous chaque graine } \\
\text { (RL); mélangé en surface sur } 10 \mathrm{~cm} \text { de } \\
\text { profondeur ((RS) }\end{array}$ \\
\hline
\end{tabular}

La dose 1 de fertilisation (Drlran et al., 1975) consistait, pour $1 \mathrm{~m}^{3}$ de tourbe, en $75 \mathrm{~g}$ de $\mathrm{N}$ (sous forme d'ammonitrate), $75 \mathrm{~g}$ de $\mathrm{P}_{2,} \mathrm{O}_{\overline{5}}$ (sous forme de superphosphate triple), $75 \mathrm{~g}$ de $\mathrm{K} . . \mathrm{O}$ (sous forme de sulfate de potassium), un $\mathrm{kg}$ de calcaire magnésien (20 p. $100 \mathrm{de} \mathrm{MgO}$ ) et $40 \mathrm{~g}$ d'un mćlange de microéléments contenant $20 \mathrm{~g}$ de sulfate ferrique, $10 \mathrm{~g}$ de sulfate de cuivre, $5 \mathrm{~g}$ de molybdate d'ammonium, $0,5 \mathrm{~g}$ de sulfate de zinc, $3,5 \mathrm{~g}$ de sulfate de manganèse et $1 \mathrm{~g}$ d'acide borique. Les variantes $1 / 2$ et 1 n'ont fait varier que la quantité globale de macroéléments (N, P, K), les quantités de calcaire et de microéléments restant constantes. Le $\mathrm{pH}$ des substrats a été ajusté à $4,5,6$ ou 7 par apport de craie broyée.

L'inoculum était une cullure mycélienne d'Hebeloma crustuliniforme sur mélangevermiculite-tourbe $(9 / 10$ de vermiculite pour $1 / 10$ de tourbe, en volume) imprégné de 
milieu nutritif. La souche utilisée a été isolée à partir d'un carpophore récolté dans unc plantation d'épicéas sur sol brun lessivé à mull mésotrophe sur limon. Dans la variante $\mathrm{L}$, l'inoculum était lavé sur un tamis à l'eau du robinet.

Les tunncls recouvrant les traitements $\mathrm{TO}$ et $\mathrm{TF}$ avaient une largeur de $1 \mathrm{~m}$ et une hauteur de $1 \mathrm{~m}$, et étaient constitués de film polyéthylène. L'arrosage y était assuré par brumisation. Dans le traitement TF, le tunnel est resté clos pendant toute la période de culture (avril à septembre). Dans le traitement TO, le tunnel a été partiellement ouvert les jours de grande chaleur, puis enlevé début août. Chaque traitement était répété 5 fois ( 5 pots, 25 plants).

En fin d'expérience (septembre), la hauteur totale de tous les plants a été mesurée et les systèmes racinaires ont été observés à la loupe à main pour quantifier le développement de la mycorhization par Hebeloma crustuliniforme, qui a été noté de 0 (pas de mycorhization visible) à 3 (mycorhizes abondantes sur toutes les racines).

\subsection{Expérience en phytotron}

Les graines de hêtre (provenance plateaux calcaires du nord-cst de la France) ont été prégermées comme précédemment et semées à raison d'une faine par cavité dans des cagettes contenant 32 godets ouvrants (« rootrainers» Spencer-Lemaire). Chaque cavité contient $150 \mathrm{~cm}^{3}$ de substrat étudié : tourbe blonde de Hollande avec différentes formules de fertilisation et différentes doses d'inoculum mélangé à la tourbe. Chaque traitement est représenté par une cagette (32 plants).

Neuf traitements de fertilisation (tableau 2) ont été combinés avec 3 doses d'inoculation : 1 p. 100,2 p. 100 et 5 p. 100 du volume de tourbe. L'inoculum. lavé, était du même type que pour l'expérience en pépinière. Il a été mélangé à la tourbe avant le remplissage des godets. Les traitements $1 / 2-111$ aux trois doses d'inoculation étaient doublés avec l'inoculum non lavé.

TABLEAU 2

Détail des 9 modalités de fertilisation de l'expérience en phytotron.

Detail of the 9 fertilization treatments in the phytotron experiment.

\begin{tabular}{|c|c|c|c|c|}
\hline & & \multicolumn{3}{|c|}{ Dose de base } \\
\hline & & $1 / 4$ & $1 / 2$ & 1 \\
\hline \multirow{3}{*}{ Equilibre $N-P_{9} O_{3}-K_{3} O$} & $111 \ldots \ldots \ldots$ & $1 / 4-111$ & $1 / 2-111$ & $1-111$ \\
\hline & 211. & $1 / 4-211$ & $1 / 2-211$ & $1-211$ \\
\hline & 121 & $1 / 4-121$ & $1 / 2-121$ & $|-12|$ \\
\hline
\end{tabular}

La dose de base 1 est la même que đans l'expérience en pépinière (équilibre $\mathrm{N}-\mathrm{P}_{2} \mathrm{O}_{5}-\mathrm{K}_{2} \mathrm{O}: 111$ ). Les ćquilibres 211 et 121 sont obtenus en doublant, respectivement, la quantité d'azote et de phosphore à partir de la dose de base. Le pH est de 4 dans tous les traitements - The basic dose 1 is the same one as in the nursery experiment $\left(N-P_{2} O_{5}-K_{2} O\right.$ balance: 111). The 211 and 121 balances are obtained by doubling the quantity of nitrogen or phosphorus, respectively, from the basic dose, $\mathrm{pH}$ is 4 in all treatments. 
Les cagettes ont été maintenues pendant 120 jours (4 mois) dans une chambre de croissance climatisée : jours longs de $16 \mathrm{~h}$ (éclairement au niveau du sommet des plants : $16000 \mathrm{lux}), 12^{\circ} \mathrm{C}$ la nuit et $20^{\circ} \mathrm{C}$ le jour, humidité atmosphérique oscillant entre 90 et 100 p. 100.

Les godets ont été ouverts à l'âge de 10, 20, 28, 38, 53, 65, 79, 92, 108 et 120 jours pour l'observation à la loupe à main de la tourbe et des racines, toujours sur la même face de la motte. Ont été notées la présence ou l'absence de mycélium d'Hebeloma crustuliniforme et l'intensité de la mycorhization (même échelle de 0 à 3 que précédemment). La hauteur de chaque plant au-dessus des cotylédons a été mesurée à 79 ct à 120 jours (fin de l'expérience).

\subsection{Expérience in virr?}

Les 9 substrats de l'expêrience on phytotron et un substrat supplémentaire constitué de lourbe pure, humidifiés à la capacité au champ, ont été étalés sur une épaisseur d'environ $5 \mathrm{~mm}$ dans les boîtes de Pétri de $90 \mathrm{~mm}$ de diamètre ( 5 bồtes par substrat). Ces boîtes ont été ensemencées par la même souche d'Hebeloma crustuliniforme (2 implants de $6 \mathrm{~mm}$ de diamètre par boîte) ou par un amas central de $2 \mathrm{ml}$ d'inoculum vermiculite-tourbe lavé ou non lavé. Elles ont été placées pendant 30 jours à l'obscurité à 25 "C.

Deux expériences successives ont été cffectuées avec les implants gélosés. Dans la première, les boîtes n'étaient pas scellées et l'humidité de la tourbe était ajustée chaque semaine par pesée et apport d'eau distillée. Dans la deuxième, les bôttes ćtaient scellées à l'aide d'un ruban adhésif pour empêcher l'évaporation.

Le développement du mycélium a été observé périodiquement sous la loupe binoculaire, sans ouvrir les boîtes. Dans le cas des expériences avec implants gélosés, il a été comparé à celui obtenu sur sable quartzeux pur stérile, imprégné d'eat stérile et maintenu stérile dans des boîtes scellées.

\section{Résultats}

\subsection{Expérience en pépinière (fig. 1)}

La mycorhization est faible dans le traitement de base (note 0,3), mais elle est considérablement augmentée (note 1,5 environ) par une réduction de la dose de fertilisation ou par augmentation de la dose d'inoculum (fig. 1 b et d). Pour la dose moyenne de fertilisation (1), la hauteur des plants n'est pas influencée par l'intensité de la mycorhization (fig. 1 b). Par contre, celle-ci compense (figure 1 d) la réduction de croissance normalement entraînée par une dose de fertilisation inférieure à 2 (DELRAN et al., 1975).

Par rapport au traitement de base, l'élévation du $\mathrm{pH}$ empêche l'infection mycorhizienne, en même temps qu'elle réduit fortement la croissance des plants (fig. 1 f). Cet oplimum de croissance du hêtre sur tourbe à $\mathrm{pH} 4$ avait déjà été mis en évidence par DeLran et al., 1975. 


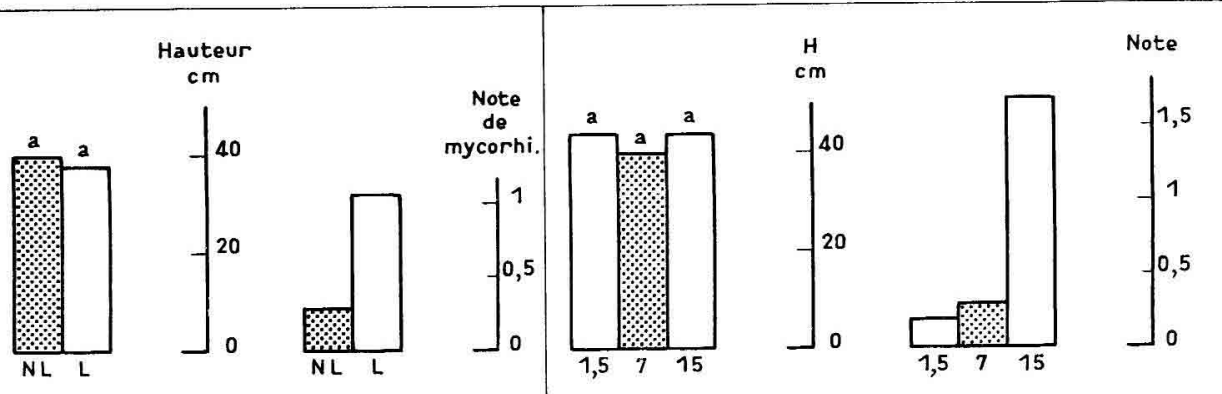

a) TYPE D'INOCULUM

b) DOSE D'INOCULUM

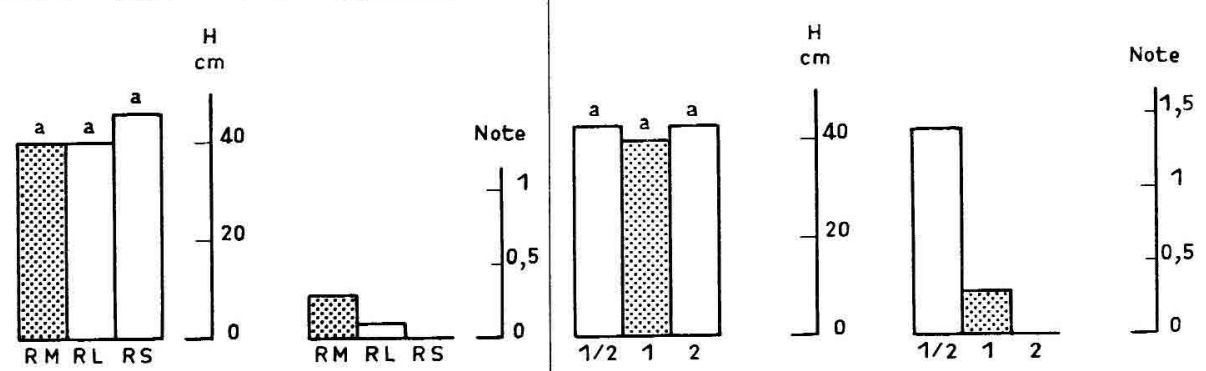

c) REPARTITION DE L'INOCULUM

d) DOSE DE FERTILISATION

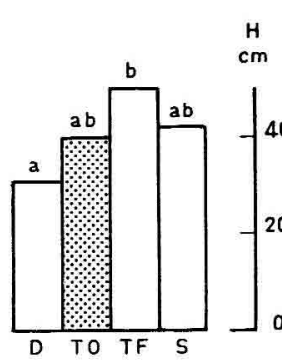

e) CLIMAT

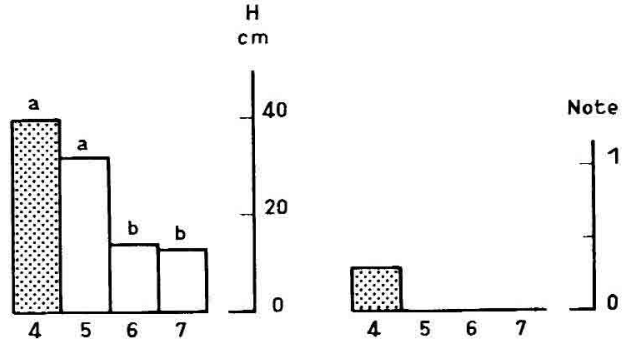

f) $\mathrm{pH}$

FIG, 1

Hauteur totale et note moyenne de mycorhization des plants dans l'expérience en pépinière.

Total height and mean mycorrhization index of the plants in the nursery experiment.

Les colonnes correspondant au traitement de base sont en grisé. Pour les hauteurs, les traitements portant la même lettre (a,b...) ne diffèrent pas significativement au risque de 5 p. 100. Voir tableau 1 pour les définitions des traitements.

The columns corresponding to the basic treatment are grey. For the heights, the treatments with the same letter $(a, b \ldots)$ are not significantly different at the $5 \mathrm{p} .100$ level. See table 1 for the definitions of treatments. 
Le mélange de l'inoculum au substrat provoque une meilleure mycorhization que sa localisation sous les graines (fig. $1 \mathrm{c}$ ), et le lavage de l'inoculum exerce un effet très positif (fig. $1 \mathrm{a}$ ).

La mycorhization ne s'est installée que dans deux climats : tunnel ouvert et serre, avec une note moyenne un peu supérieure dans ce dernier cas (fig. $1 \mathrm{e}$ ).

\subsection{Expérience en phytotron}

- Développement des plants. La levée des graines est terminée dès le $10^{\circ}$ jour, avec les cotylédons étalés. Les premières racines courtes d'ordre 2 et 3 apparaissent dès le $28^{\prime \prime}$ jour. Le $38^{\prime \prime}$ jour, les deux premières feuilles sont étalées sur tous les plants. A partir du $53^{\circ}$ jour, la pousse s'allonge et de nouvelles feuilles apparaissent et le nombre des racines courtes augmente rapidement avec le développement du système racinaire. Les cotylédons sèchent et tombent à partir du 92" jour.

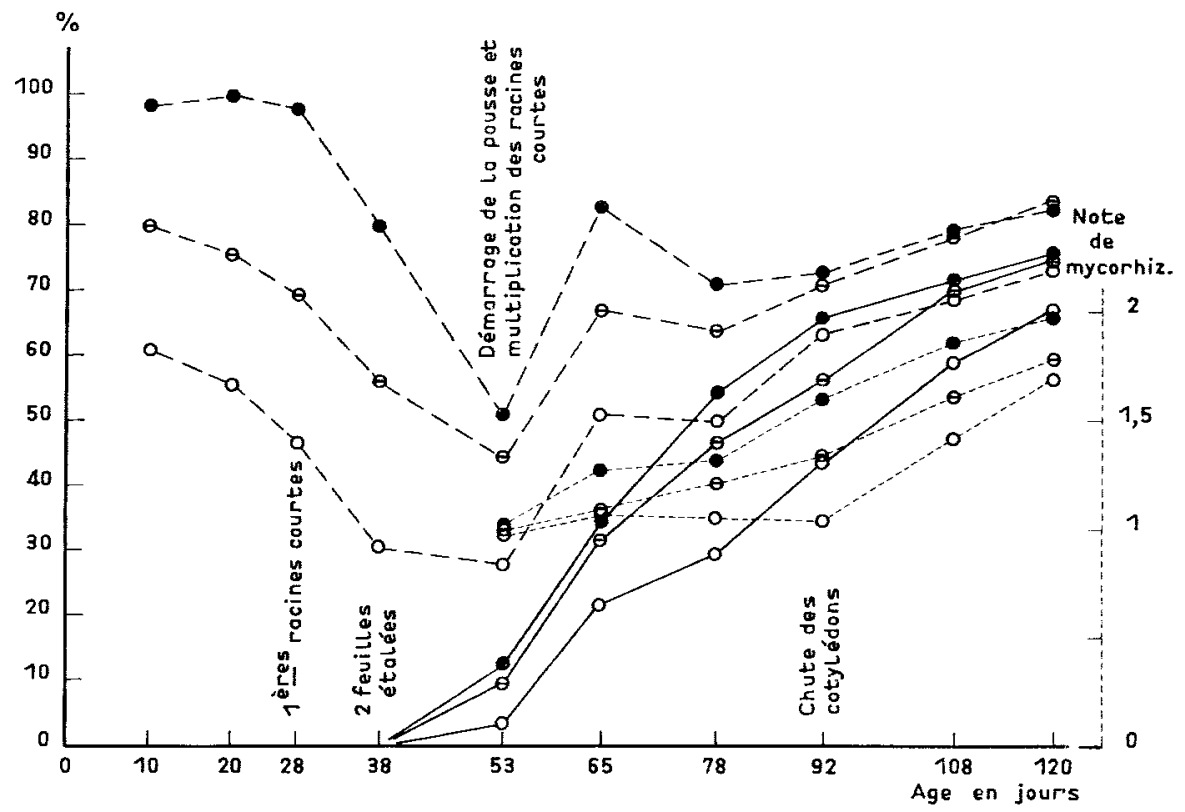

Fig. 2

Moyemes des 9 modalités de fertilisation.

Mean values of the 9 fertilization treatments.

Pourcentage de godets présentant du mycélium (tirets), pourcentage de plants mycorhizés (trait continu) et note moyenne de mycorhization des plants mycorhizés (pointillés) en fonction du temps et de la dose d'inoculum $(0: 1$ p. $100 \mathrm{v} / \mathrm{v} ; \Theta: 2$ p. 100; $: 5$ p. 100).

Percent of containers with mycelitum (broken line), percent of mycorrlized plants (solid line) and mean mycorrhization index of the mycorrhized plants (dotted line) related to time and inoculam dose $(0: 1$ p. $100 \mathrm{v} / \mathrm{v} ; \Theta: 2 \mathrm{p} .100 ; \bullet: 5 \mathrm{p}: 100)$. 
- Installation de la mycorhization (fig. 2). Les premières mycorhizes apparaissent entre le $38^{\circ}$ et le $53^{\prime \prime}$ jour, puis le pourcentage de semis mycorhizés augmente, rapidement au début puis de mojns en moins vite. 11 continue d'augmenter en fin d'expérience (120 jours), mais la forme des courbes suggère qu'il n’atteindra pas 100 p. 100 . Parallèlement, la note moyenne de mycorhization des semis mycorhizés augmente régulièrement à partir du 78" jour. Elle n'est appréciable que 20 jour's après l'apparition des premières mycorhizes. WILSON et HARLEY (1983) observent lá même succession d'événements sur des semis de hêtre cultivés en serre sur un sol forestier.

-- Développement du mycélium (fig. 2). Dès le 10" jour, du mycélium est visible sur la plupart des mottes. Dans tous les cas, il sort des fragments de vermiculite de l'inoculum, avec une extension maximale de 1 à $2 \mathrm{~mm}$. La fréquence du mycélium dans les godets décroît ensuite jusqu'au 53" jour, qui marque le début de la mycorhization, puis augnente jusquà la fin des observations, restant toujours supéricurc au pourcentage de plants mycorhizés. Le mycélium observé est alors en majeure partic autour des mycorhizes ou des racines courtes non mycorhizées, sans lien apparent avec la vermiculite.

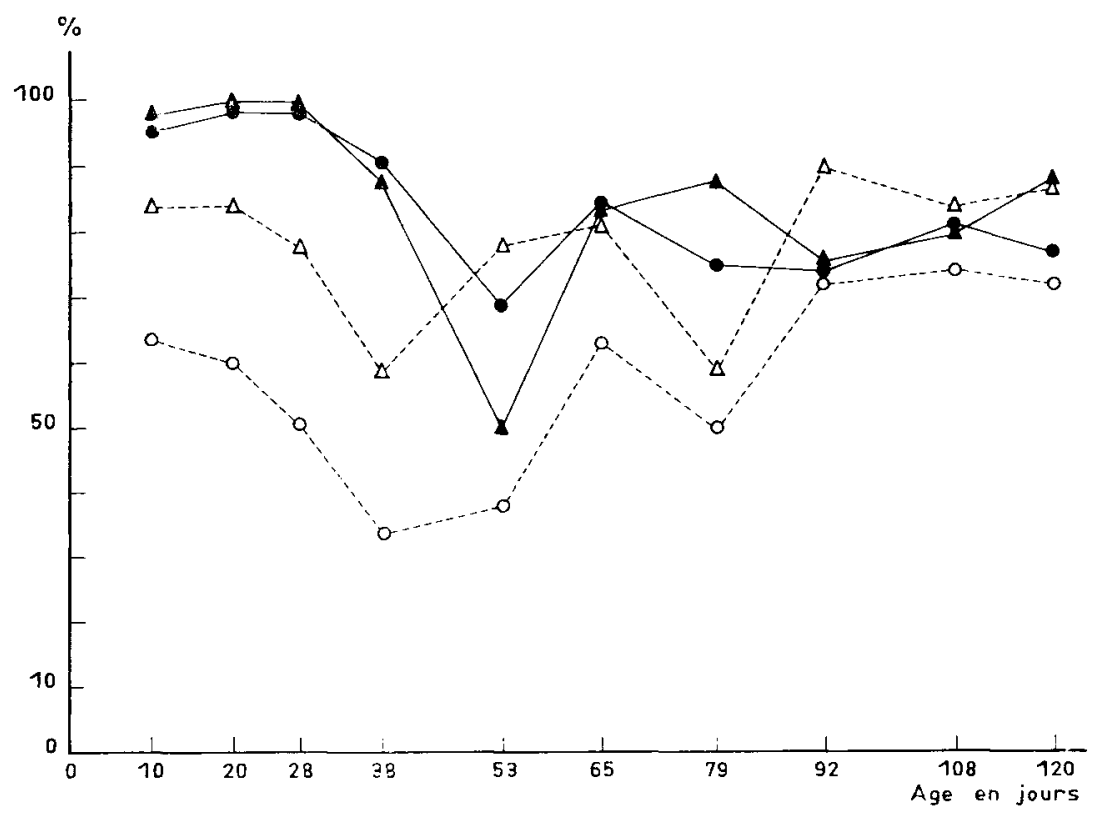

Fig. 3

Pourcentage de godets présentamt du mycélium en fonction du temps, de la dose d'inculum et da lavage de l'inoculum.

Percent of containers with mycelium related to time, inoculum dose and washing of inoculum.

$O$ et $\bullet$ : inoculum lavé - washed inoculum.

$\Delta$ et $\Delta$ : inoculum non lavé - non-w'ashed inoculum.

$O$ et $\triangle$ : faible dose d'inoculum $(1$ p. $100 \mathrm{v} / \mathrm{v})-$ low dose of inoculum $(1 \mathrm{p} .100 \mathrm{v} / \mathrm{v})$.

- et $\wedge$ : forte dose d'inoculum $(5 \mathrm{p} .100 \mathrm{v} / \mathrm{v})$ - high dose of inoculum $(5 \mathrm{p} .100 \mathrm{v} / \mathrm{v})$. 
- Effet de la dose dinoculum (fig. 2). Les trois paramètres précédents varient dans le même sens que la dose d'inoculum initialement mélangée à la tourbe, avec, en fin d'expérience, moins de différence entre les doses 2 p. 100 et 5 p. 100 , qu'entre les doses 1 p. 100 et 2 p. 100 , sauf cependant pour la note moyenne de mycorhization.

- Effet du lavage de l'inoculum. Lorsque la dose d'inoculum est faible (1 p. 100), le lavage diminue la survie du mycélium (fig. 3), mais n'a pas d'influence sur la mycorhization. Lorsque la dose d'inoculum est forte (5 p. 100), le lavage n'a aucun effet sur les 3 paramètres mesurés.

- Effet de la fertilisation (fig. 4, 5,6 et 7). Les résultats présentés ne concernent que la plus faible dose d'inoculum ( 1 p. $100 \mathrm{v} / \mathrm{v})$, pour laquelle l'effet de la fertilisation est le plus marqué. Pour une dose globale faible $(1 / 4$, fig. 4 et 5$)$, l'équilibre $\mathrm{N}-\mathrm{P}_{2} \mathrm{O}_{5},-\mathrm{K}_{2} \mathrm{O}$ a un effet très marqué sur les trois paramètres étudiés, toujours dans $1 \mathrm{e}$ même sens (par ordre de valeur décroissante des paramètres : 121, 211, 111). Pour une dose globale forte (1, fig. 6 et 7 ), l'effet est encore très marqué, mais en sens inverse : l'équilibre de base 111 donne les meilleurs résultats. Pour la dose globale

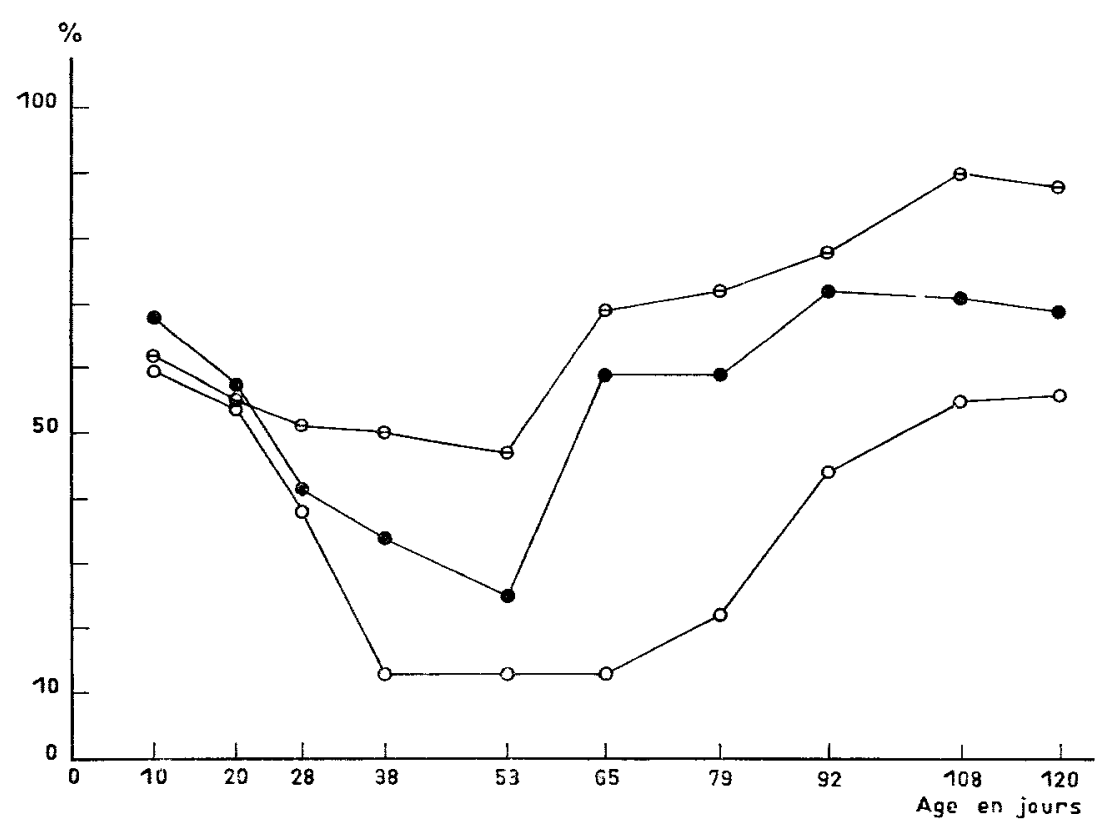

FIG. 4

Pourcentage de godets présentant du mycélium en fonction du temps et de l'équilibre $\mathrm{N}-\mathrm{P}_{2} \mathrm{O}_{i}-K_{3} \mathrm{O}$, pour la plus faible dose d'inoculum et la plas faible dose de fertilisation $(1 / 4)$.

Percent of containers with mycelium related to time and to $\mathrm{N}-\mathrm{P}_{2} \mathrm{O}_{5}-\mathrm{K}_{3} \mathrm{O}$ balance for the lower inoculum dose and the lower fertilization (1/4).

0 : équilibre 111 - 111 balance.

- : équilibre 211 - 211 balance.

$\Theta$ : équilibre $121-121$ balance. 


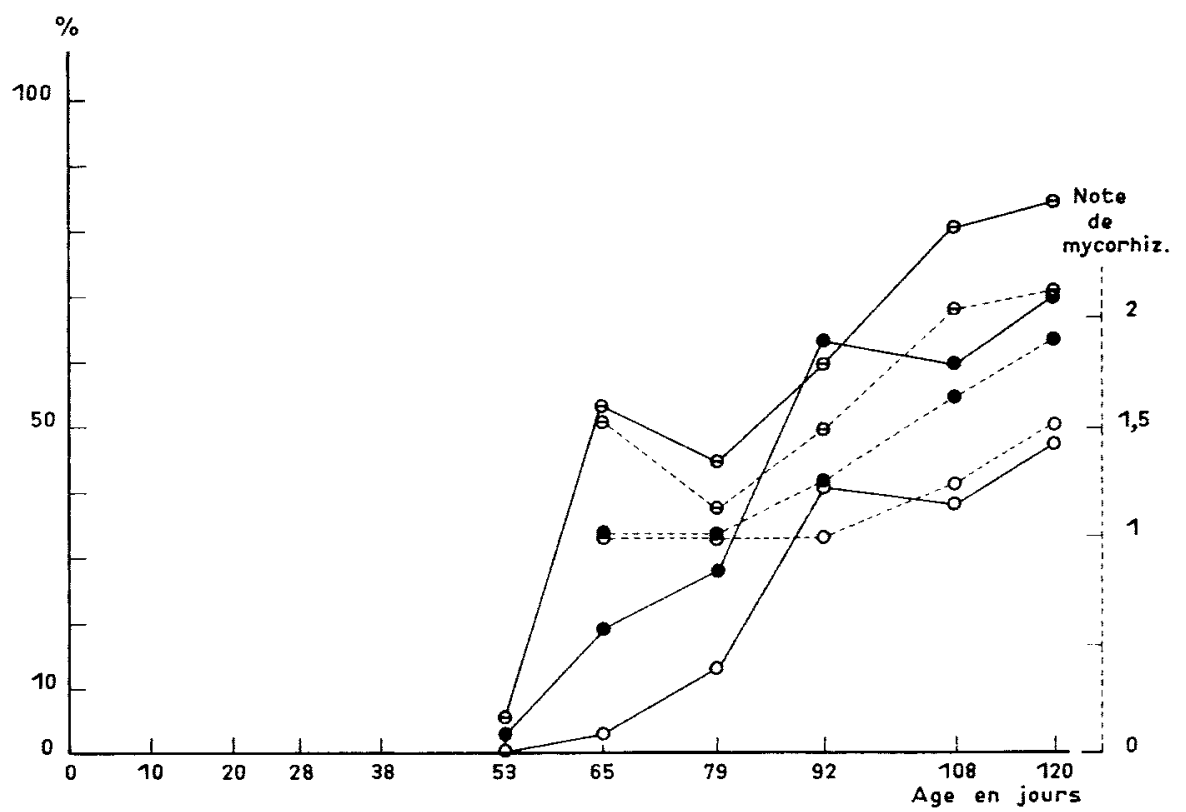

FIG. 5

Pourcentage de plants mycorhizés (traits continus) et note moyenne de mycorhization des plants mycorhizés (traits pointillés) en fonction du temps et de l'équilibre $N-P_{s} O_{i}-K_{*} O$ pour la plus faible dose d'inoculum et la plus faible dose de fertilisation (1/4).

Percent of mycorrhized plants (solid line)

and mean mycorrhization index of the mycorrhized plants (dotted line) related to time and to $N-P_{2} O_{3}-K_{2} O$ balance, for the lower inoculum dose and the lower fertilization $(1 / 4)$.

$\bigcirc$ : équilibre 111 - 111 balance.

- : équilibre 211 - 211 balance.

$\Theta$ : équilibre $121-121$ balance.

\section{Tableau 3}

Pourcentage de plants mycorhizés et note moyenne de mycorhization des plants inycorhizés (entre parenthèses) en fonction de la dose de fertilisation et de l'équilibre $\mathrm{N}-\mathrm{P}_{2} \mathrm{O}_{5}-\mathrm{K}_{8} \mathrm{O}$, pour la plus faible dose d'inoculum $(1 \mathrm{p} .100 \mathrm{v} / \mathrm{v})$.

Percentage of mycorrhizal plants and mean mycorrhization note (in brackets) of the mycorrhized plants related to fertilization dose and $\mathrm{N}-\mathrm{P}_{2} \mathrm{O}_{5}-\mathrm{K}_{2} \mathrm{O}$ balance, for the lowest inoculum dose $(I$ p. $100 \mathrm{v} / \mathrm{v})$.

\begin{tabular}{|c|c|c|c|c|}
\hline & & \multicolumn{3}{|c|}{ Dose de base } \\
\hline & & $1 / 4$ & $1 / 2$ & 1 \\
\hline \multirow{3}{*}{ Equilibre $\mathrm{N}-\mathrm{P}_{2} \mathrm{O}_{i}-\mathrm{K}, \mathrm{O}$} & $111 \ldots \ldots \ldots$ & $47(1,5)$ & $75(2)$ & $84(1,9)$ \\
\hline & $211 \ldots \ldots \ldots$ & $69(1,9)$ & $53(1,9)$ & $53(1)$ \\
\hline & $121 \ldots \ldots \ldots$ & $84(2,1)$ & $74(1,7)$ & $47(1,3)$ \\
\hline
\end{tabular}




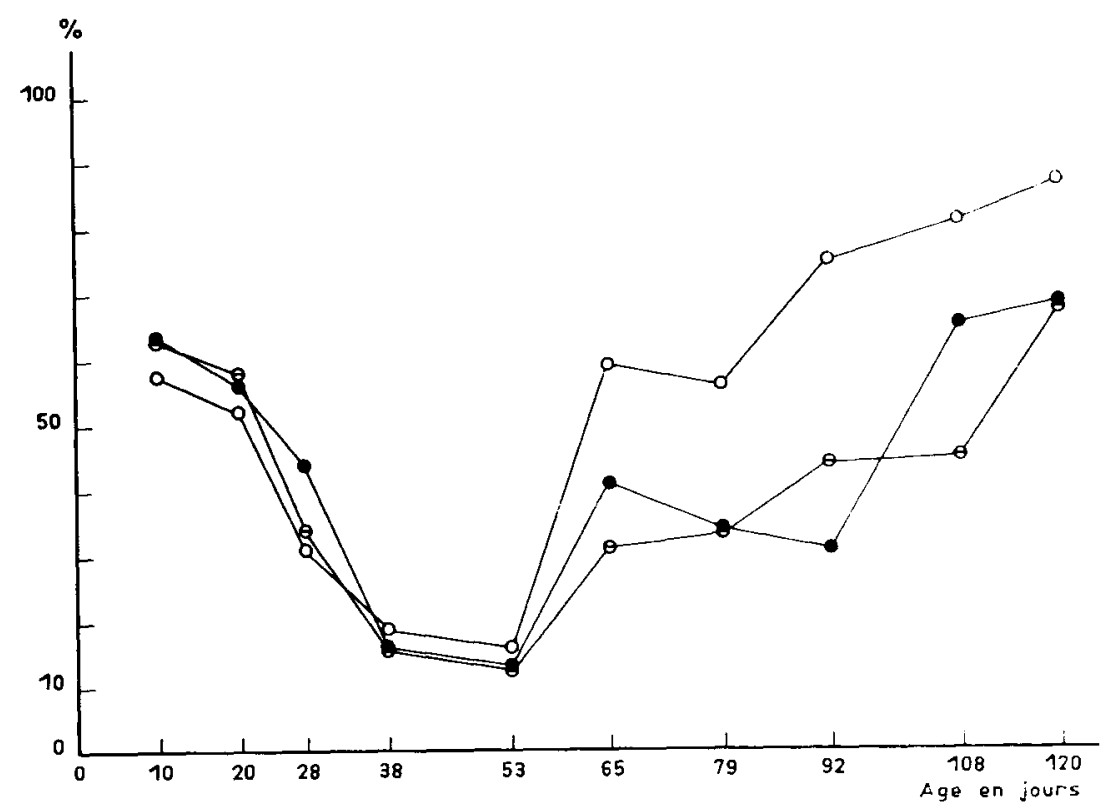

FIG. 6

Pourcentage de godets présentant du mycélium en fonction du temps et de l'équilibre $\mathrm{N}-\mathrm{P}_{z} \mathrm{O}_{5}-\mathrm{K}_{2} \mathrm{O}$ pour la plus faible dose d'inoculum et la plus forte dose de fertilisation (1).

Percent of containers with mycelium related to time and to $\mathrm{N}-\mathrm{P}_{2} \mathrm{O}_{5}-\mathrm{K}_{2} \mathrm{O}$ balance, fot the lower inoculum and the highest fertilization (1).

$O$ : équilibre $111-111$ balance.

- : équilibre 211 - 211 balance.

$\Theta$ : équilibre 121 - 121 balance.

intermédiaire (1/2), aucun cfict n'apparait nettement. Parmi les 9 formules de fertilisation, la meilleure mycorhization en fin d'expérience (tableau 3) est obtenue indifféremment avec 1/4-121 ou 1-111, aussi bien en pourcentage de plants mycorhizés qu'en intensité de la mycorhization. On remarque par ailleurs, que pour les doses extrêmes ( $1 / 4$ et surtout 1$)$ les différences de fréquence du mycélium n'apparaissent que tardivement, suggérant que la composition minérale du substrat est moins la cause première des différences observécs que, indirectement, l'état physiologique de la plante et la composition de la rhizosphère, du fait de différences de nutrition minérale.

- Croissance des plants. Le fait de ne pas laver l'inoculum réduit la croissance, surtout pour la dose la plus forte $(5 \mathrm{p} .100 \mathrm{v} / \mathrm{v}): 10,9 \mathrm{~cm}$ contre $15,5 \mathrm{~cm}$ de hauteur au-dessus des cotylédons à 120 jours, et entraîne la mort de 25 p. 100 de plants, alors que la mortalité n'excède jamais 5 p. 100 dans les traitements avec inoculum lavé, quelle que soit la dose. La fertilisation a un effet très marqué sur la croissance (tableau 4). Une dose de base élevée a un effet positif auquel s'ajoute l'effet positif du renforcement en azote (équilibre 211). La comparaison avec le tableau 3 montre l'absence de relation entre le niveau d'infection mycorhizienne et la croissance. 


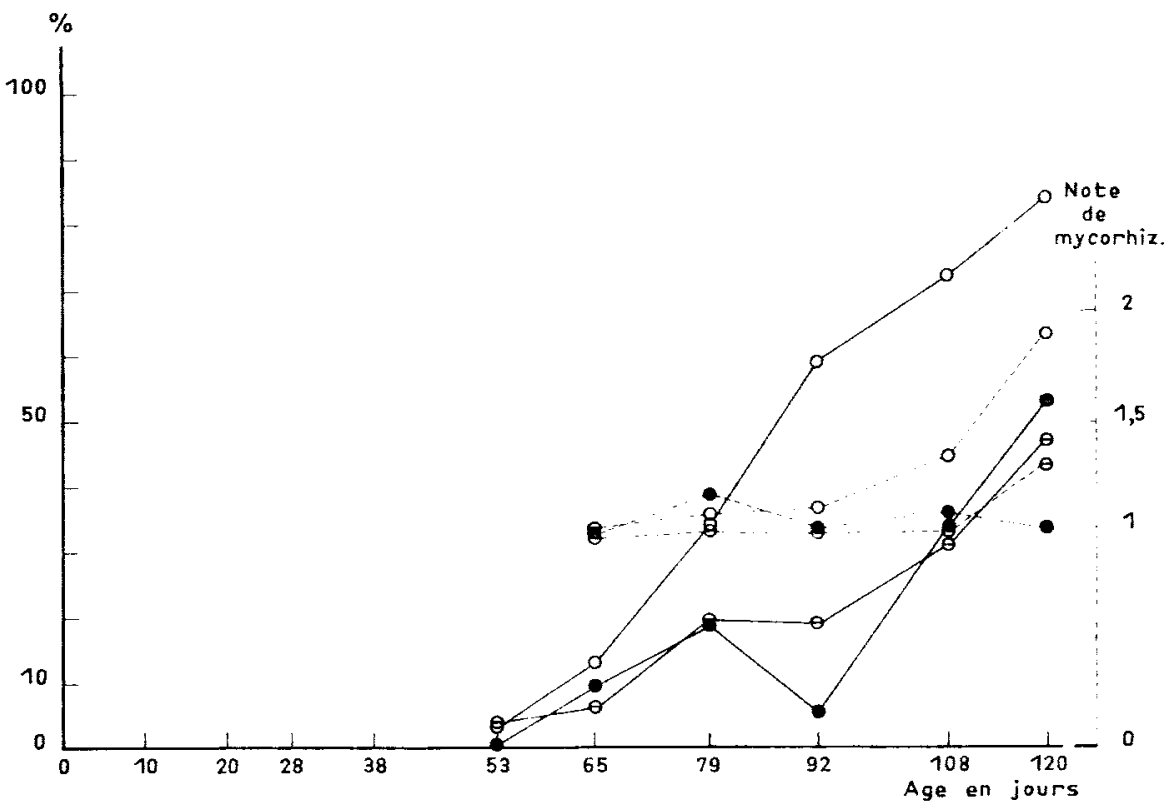

Fig. 7

Pourcentage de plants mycorhizés (traits continus)

et note moyenne de mycorhization des plants mycorhizés (traits pointillés)

en fonction du temps et de l'équilibre $N-P_{s} O_{i}-K_{s} O$ pour la plus faible dose d'inoculum et la plus forte dose de fertilisation (1).

Percent of mycorrhized plants (solid line)

and mean mycorrhization index of the mycorrhized plants (dotted line)

related to time and to $\mathrm{N}-\mathrm{P}_{2} \mathrm{O}_{3}-\mathrm{K}_{2} \mathrm{O}$ balance,

for the lower inoculum dose and the highest fertilization (1).

0 : équilibre 111 - 111 balance.

- : équilibre 211 - 211 balance.

$\Theta$ : équilibre 121 - 121 balance.

\section{TABLEAU 4}

Hauteur des plants en cm au-dessus des cotylédons le $79^{\circ}$ jour et le $120^{\circ}$ jour (entre parenthèses) en fonction de la dose de fertilisation et de l'équilibre $N-P_{s} O_{s}-K_{s} O$, pour la plus faible dose d'inoculum (I p. $100 \mathrm{v} / \mathrm{v})$.

Height of the plants above the cotyledons on 79 th day and on 120th day (in brackets) in $\mathrm{cm}$ related to fertilization dose and $\mathrm{N}-\mathrm{P}_{2} \mathrm{O}_{s}-\mathrm{K}_{2} \mathrm{O}$ balance for the lowest inoculum dose $(1 \mathrm{p} .100 \mathrm{v} / \mathrm{v})$.

\begin{tabular}{|c|c|c|c|c|}
\hline & & \multicolumn{3}{|c|}{ Dose de base } \\
\hline & & $1 / 4$ & $1 / 2$ & 1 \\
\hline Equilibre $N-P_{2} \mathrm{O}_{-}-\mathrm{K}_{2} \mathrm{O}$ & $\begin{array}{l}111 \ldots \ldots \\
211 \ldots \ldots \\
121 \ldots \ldots\end{array}$ & $\begin{array}{l}11,2(11,8) \\
13,3(14,3) \\
11,5(11,5)\end{array}$ & $\begin{array}{l}12,9(14,2) \\
15,1(16,4) \\
11,6(13,5)\end{array}$ & $\begin{array}{l}13,1(14,7) \\
15,1(17,1) \\
11,8(13,4)\end{array}$ \\
\hline
\end{tabular}




\subsection{Expérience in vitro}

Dans les expériences avec implants gélosés, le mycélium des témoins sur quartz se développe jusqu'à atteindre une extension radiale de 5 à $10 \mathrm{~mm}$ en 10 jours, à partir des réserves nutritives de l'implant. Sur la tourbe, le mycélium ne se développe pas ou n'atteint qu'une extension radiale de 1 à $2 \mathrm{~mm}$. Le pourcentage d'implants ayant ainsi émis du mycélium après 30 jours d'incubation est très variable et ne permet aucune conclusion, sinon que la tourbe inhibe fortement la croissance d'Hebeloma crustuliniforme.

L'expérience avec inoculum vermiculite-tourbe montre également une très faible croissance du mycélium sur la tourbe (1 à $2 \mathrm{~mm})$, mais une meilleure survie qu'avec les implants gélosés. Le taux de survie est cependant généralement aussi bon ou meilleur avec l'inoculum lavé plutôt que non lavé. II n'apparaît aucune relation simple entre le type de fertilisation et le développement ou la survie du mycélium. On remarque seulement que la croissance est totalement inhibée sur la tourbe pure non fertilisée, que l'inoculum soit lavé ou non. Un apport d'éléments minéraux est donc favorable.

\section{Discussion}

L'expérience en phytotron nous montre la dynamique de la mycorhization dans des conditions climatiques régulières et proches de l'optimum. Dès les premiers jours, le mycélium croît faiblement à partir des fragments de vermiculite à l'intérieur desquels il avait été protégé lors du mélange de l'inoculum à la tourbe. L'abondance du mycélium dans le substrat dépend alors étroitement de la concentration initiale en inoculum : un fragment de vermiculite, s'il abrite du mycélium viable, ne peut donner qu'un volume de quelques $\mathrm{mm}^{3}$ contenant le champignon. Le mycélium cesse ensuite de croître et commence à disparaître dès le $20^{*}$ jour. Cette première phase dépend peu du type de fertilisation (ce que corroborent les expériences in vitro; il semble cependant qu'un apport minimum d'éléments minéraux soit favorable à la survie du mycélium). Le lavage de l'inoculum, en l'appauvrissant en milieu nutritif résiduel, ne réduit le développement et la survie du mycélium que pour les faibles doses d'inoculation ( 1 p. $100 \mathrm{v} / \mathrm{v}$ ). Le facteur limitant l'expansion du mycélium dans le substrat et sa survie est donc plus lié à la tourbe elle-même (facteurs physico-chimiques ou microbiologiques défavorables comme le montre l'expérience in vitro avec les implants gélosés) qu'aux réserves nutritives de l'inoculum. L'interaction entre l'effet du lavage et la dose d'inoculum est probablement due à un autre facteur non contrôlé : effet de la microflore saprophytique utilisant les réserves nutritives de l'inoculum (MARX, 1980).

Cette phase de décroissance de la présence mycélienne irait probablement jusqu'à la disparition complète en l'absence de plante-hôte. Mais en présence de l'hôte, les premières racines courtes apparaissent vers le 30" jour, suivies environ 10 jours après par les premières mycorhizes.

Le nombre de plants mycorhizés augmente alors rapidement, mais l'intensité de la mycorhization de ces plants ne commence à croître que 20 à 30 jours plus tard. Ces deux étapes correspondent respectivement à l'apparition des points d'infection 
primaire de racines courtes isolées à partir du mycélium issu de l'inoculum, puis à la multiplication des points d'infection secondaire à partir des mycorhizes déjà formées, de leur mycélium frangeant et du mycélium rhizosphérique. ChILvers \& Gust (1982) ont déjà décrit ce phénomène sur les semis d'eucalyptus.

Dès le début de la mycorhization, la fréquence du mycélium dans les godets, qui avait diminué jusqu'alors, augmente brutalement et continue à croître jusqu'à la fin des observations. Il ne s'agit plus de mycélium issu de la vermiculite, mais de mycélium lié aux mycorhizes ou situé au voisinage immédiat de racines courtes non infectées. Le pourcentage de godets présentant du mycélium reste supérieur au pourcentage de plants mycorhizés (10 p. 100 environ), l'écart restant constant à partir du 90* jour. Comme la proportion de plants mycorhizés augmente, cela prouve que de nouveaux plants continuent en permanence à capter du mycélium dans leur rhizosphère à partir de l'inoculum déclinant et à assurer sa survie et sa croissance par l'intermédiaire de leurs exsudats racinaires, avant de contracter à leur tour l'association symbiotique. La figure 8 schématise les différentes phases de l'infection mycorhizienne telle que nous venons de la décrire.

Les infections primaires ne peuvent avoir lieu que si le relais est assuré à temps entre la phase 1 et la phase 2. En pratique, il faut donc que la durée de recouvrement de ces 2 phases soit la plus longue possible, soit en prolongeant la survie de l'inoculum (phase 1), soit en hâtant l'apparition de racines courtes réceptives à l'infection mycorhizienne ou capables d'assurer la croissance rhizosphérique du champignon (phase 2). Une autre solution consiste à inoculer après le semis, lorsque les premières racines courtes apparaissent (LAPEYrIE, 1983). Cette technique complique cependant les opérations en pépinière.

Nos résultats montrent que la phase 1 dépend davantage de l'inoculum lui-même que de la fertilisation de la tourbe. Les améliorations sont done à rechercher d'une part dans la protection et l'inclusion du mycélium dans un substrat autre que la vermiculite, par exemple dans un gel (LE TACON et al., 1983,) et d'autre part dans l'optimisation du stade de développement et de l'infectivité du mycélium au moment de l’inoculation (LAPEYRie, 1983 ; LitTKE et al., 1980). La phase 2, par contre, dépend étroitement de la fertilisation, par l'intermédiaire de la nutrition minérale de la plante qui modifie sa composition rhizosphérique et sa réceptivité à la symbiose. A cet égard, l'intéraction entre la dose totale de fertilisation et l'équilibre azote-phosphore est particulièrement marquée.

On remarque également qu'à tous les stades du processus les résultats dépendent de la densité initiale de l'inoculum dans le substrat. Chaque élément d'inoculum (particule de vermiculite) ayant un faible rayon d'action, le nombre et la répartition homogène de ces éléments augmentent en effet la probabilité de rencontre du mycélium avec des racines courtes : ce sont les racines qui vont à l'inoculum et non pas le contraire. Tout facteur accélérant le développement racinaire ou le concentrant dans le volume occupé par l'inoculum (conteneurs) sera donc favorable, à condition yu'il ne réduise pas simultanément la réceptivité individuelle des racines courtes.

Face à ces nombreuses interactions el à la complexité du phénomène, l'expérimentation en vraie grandeur reste nécessaire pour l'optimisation des conditions d'inoculation. L'expérience en pépinière a confirmé l'importance de la dose d'inoculum, de son mélange homogène au substrat, du niveau de fertilisation. Par contre, elle révèle un effet très positif du lavage de l'inoculum sur l'établissement de la mycorhization, contredisant en partic l'expérience en phytotron. 


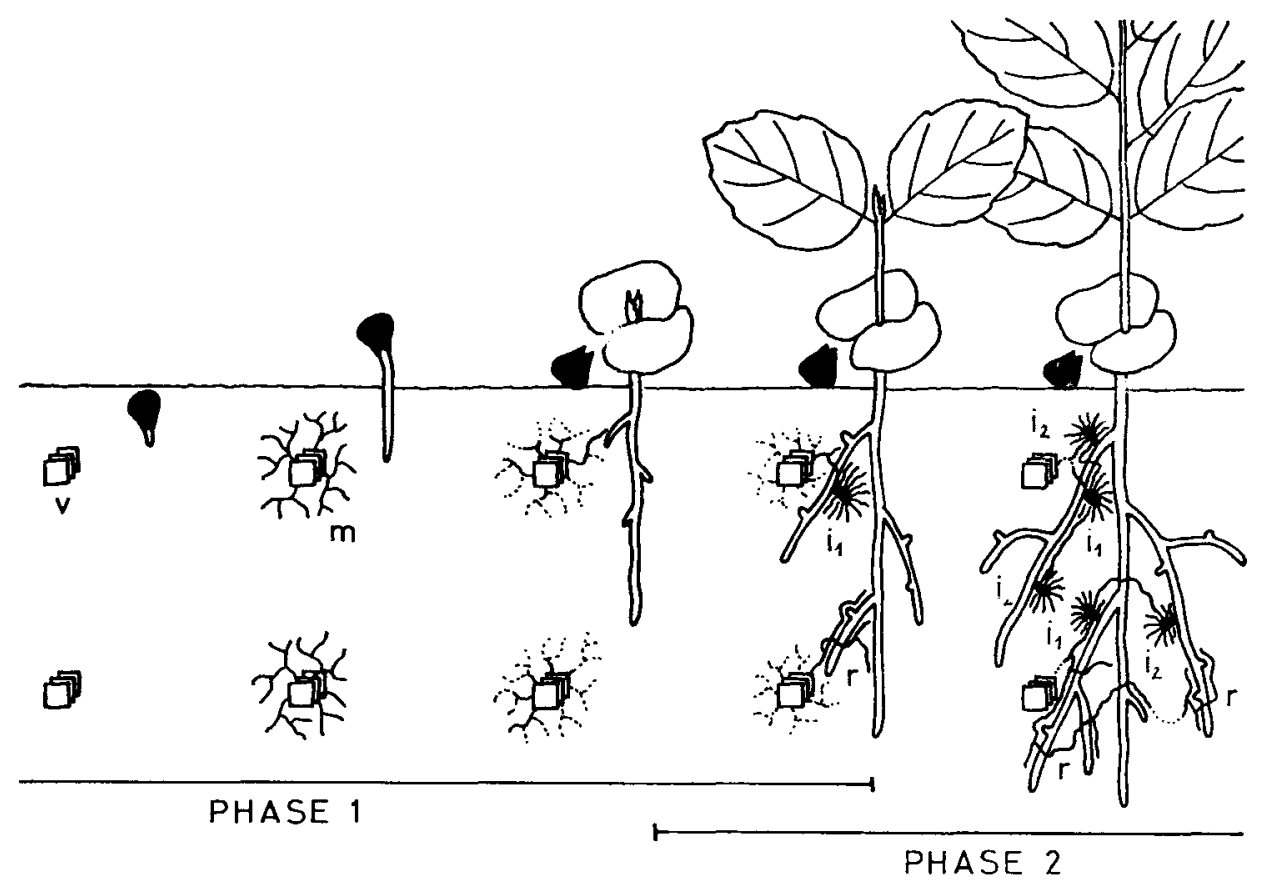

FIG, 8

Schéna de la mycorhization du hêtre par Hebeloma crustuliniforme à partir de l'inoculum vermiculite.

Diagram of the mycorrhization of beech by Hebeloma crustuliniforme from vermiculite inoculum.

Phase 1 : émergence puis déclin et mort du mycélium (m) contenu dans la vermiculite (v). Phase 2 : survie et croissance rhizosphyérique du champignon ( $r$, infection primaires $\left(i_{1}\right)$ puis secondaires $\left(i_{2}\right)$.

Phase 1: outgrowth and decay of mycelium ( $m$ ) included in vermiculite (v). Phase 2 : rhizospheric survival and growth of the fungus $(r)$, primary $\left(i_{1}\right)$ then secondary $\left(i_{*}\right)$ infections.

En pratique et compte tenu de ces résultats, il semble que de bonnes conditions d'obtention de plants de hêtre mycorhizés par Hebeloma crustuliniforme sur tourbe fertilisée soient les suivantes :

- $\mathrm{pH} 4$;

- niveau de fertilisation faible (dose 1/4) avec un renforcement en phosphore (121) ou moyenne (dose 1) avec l'équilibre de base (111);

— inoculum mélangé à la tourbe, lavé ;

- forte dose d'inoculum du type vermiculite (au moins 10 p. 100 du volume de la tourbe) ou dose plus faible d'un inoculum mieux protégé (gel) ;

- concentration du système racinaire dans un volume restreint (conteneurs ou forte densité de semis sur un Jit de tourbe d'épaisseur inférieure à $20 \mathrm{~cm}$ ). 


\section{Remerciements}

Nous remercions l'Office National des Forêts, grâce au financement duquel ces recherches ont pu être effectuées.

\section{Summary}

Limiting factors and dynamics of controlled mycorrhization of Fagus silvatica Lin. by Hebeloma crustuliniforme (Bull. ex St-Amans) Quél. on fertilized peat

The conditions of mycorrhization of beech (Fagus silvatica Lin.) by Hebeloma crustuliniforme (Bull. ex St-Amans) Quél. on fertilized peat have been studied in the nursery, in a growth chamber and in vitro. Two phases can be distinguished after seeding and mixing the inoculum (mycelium in vermiculite) with the peat : the first phase is a period of survival then decay of mycelium around vermiculite particles. The second phase is a colonization of the rhizosphere by the fungus; simultaneously, primary and secondary infections occur. Fungal survival during the first phase is independant of peat fertilization (total dose of fertilizer and nitrogen/phosphorus ratio), which is very important during the second phase. These results have led to the conception of an efficient technic for controlled mycorrhization in fertilized peat nurseries.

\section{Références bibliographiques}

Chilvers G., Gust J.W., 1982. The development of mycorrhizal populations on pot-grown seedlings of Eucalypus St-Johnii R.T. Bak. Newphytol., 80, 677-699.

Dleran S., Garbaye J., J.J: Tacon F., 1975. Production rapide de plants feuillus sur tourbe fertilisée. Nouveaux résultats. R.F.F., 27 (6), 437-448.

Delran S., Garbaye J., Kabre A., Le Tacon F., Perrin R., 1982. Lat production mapide de plants feuillus sur tourbe fertiliséc. Les problèmes microbiologiques posés par la réutilisation de la tourbe pendant plusieurs années consécutives. R.F.F., 35 (4), 314-326.

Garbaye J., 1983. Effet du champignon mycorhizien Hebeloma crustuliniforme sur la croissance du chêne et du hêtre. R.F.F., 35 (1), 21-26.

LAinez J., 1981. La mycorhization contrôlée du hêtre et du chêne en Lorraine. Premiers résultats en pépinière sur tourbe. Mémoire de 3" année, E.N.I.T.E.F., 80 p.

LAPEYRIE F., 1983. Recherches préliminaires sur le rôle des ectomvcorhizes dans limplantation d'Eucalyptus delegatensis en France : conditions d'inoculation des plants ef comportement des partenaires en présence de calcaire. Thèse de Docteur-Ingénicur, Université Claude Bernard, Lyon I, $156 \mathrm{p}$.

Le Tacon F., Jung G., Micheior P., Muginier M., 1983. Efficacité en pépinière forestière d'un inoculum de champignon ectomycorhizien produit en fermenteur ef inclus dans une matrice de polymères. Alm. Sci. For., 40 (2), 165-176.

LitTKe W.R., Bledsof C.S., Madkarni N.M., Edmonds R.L., 1980. Technique for rapid mycorrhizal colonisation of container-grown Douglas-fir by Hebeloma crustuliniforme. Soil. Biol. Biochem., 12, 575-578.

MARX D., 1980. Ectomycorrhizal fungus inoculations : a tool for improving forestation practice. In : "Tropical Mycorrhiza Research», Mikola editor, Oxford University Press, 13-71.

Wilhelm M.E., 1983. Mycorhization du chêne et du hêtre. Maîtrise en pépinière sur tourbe et évolution après plantation. Mémoire de 3" année, E.N.I.T.F.F., 82 p.

Wilson J.W., Harley J.L., 1983. The development of mycorrhiza on seedlings of Fagus silvatica L. New Phytol., 95, 673-695. 